\title{
Markowitz-Type Heuristics for Computing Jacobian Matrices Efficiently
}

\author{
Andreas Albrecht, Peter Gottschling ${ }^{1}$, and Uwe Naumann ${ }^{2}$ \\ 1 Department of Computer Science, University of Hertfordshire \\ \{A.Albrecht, P.1.Gottschling\}oherts.ac.uk \\ 2 Mathematics and Computer Science Division, Argonne National Laboratory \\ naumannemcs. anl.gov
}

\begin{abstract}
We consider the problem of accumulating the Jacobian matrix of a nonlinear vector function by using a minimal number of arithmetic operations. Two new Markowitz-type heuristics are proposed for vertex elimination in linearized computational graphs, and their superiority over existing approaches is shown by several tests. Similar ideas are applied to derive new heuristics for edge elimination techniques. The well known superiority of edge over vertex elimination can be observed only partially for the heuristics discussed in this paper. Nevertheless, significant improvements can be achieved by the new heuristics both in terms of the quality of the results and their robustness with respect to different tiebreaking criteria.
\end{abstract}

\section{Introduction}

Consider the nonlinear vector function $F: \mathbb{R}^{2} \rightarrow \mathbb{R}^{2}$ that is given by the following sequence of scalar assignments:

$$
v_{1}=v_{-1} v_{0} ; v_{2}=\sin \left(v_{1}\right) ; v_{3}=v_{1} v_{2} ; v_{4}=\cos \left(v_{3}\right) ; v_{5}=\exp \left(v_{3}\right) .
$$

For simplicity, all variables carry a unique index. There are $n=2$ independent, $p=3$ intermediate, and $m=2$ dependent variables. Mathematical functions that are implemented as computer programs written in an imperative programming language such as $\mathrm{C}$ or Fortran can always be decomposed to meet this requirement. The structure of such computations can be visualized by a directed acyclic graph (dag) $G=(V, E)$ as shown in Figure 1. If one assumes that jointly continuous local partial derivatives

$$
c_{j, i} \equiv \frac{\partial}{\partial v_{i}} \varphi_{j}\left(v_{k}\right)_{k \prec j}, \quad j=1, \ldots, p+m
$$

of the elemental functions (e.g., *, sin, $\cos , \exp )$ with respect to its arguments exist in some neighborhood of the current point, the corresponding numerical values, computed as

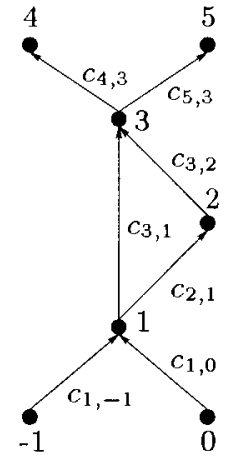

Fig. 1. $F$

$$
c_{1,-1}=v_{0} ; c_{1,0}=v_{-1} ; c_{2,1}=\cos \left(v_{1}\right) ; c_{3,1}=v_{2} ; c_{3,2}=v_{1}
$$




$$
c_{4,3}=-\sin \left(v_{3}\right) ; c_{5,3}=v_{5} \quad,
$$

can be attached to the edges in the dag. The notation $k \prec j$ is used to indicate that $v_{k}$ is an argument of $\varphi_{j}$. Thus one gets the linearized computational graph (or c-graph) $G=(V, E)$ of $F$ as displayed in Figure 1. Its vertices $V=X \cup Z \cup Y$, where $X=\{1-n, \ldots, 0\}, Z=\{1, \ldots, p\}$, and $Y=\{p+1, \ldots, p+m\}$, are numbered consistently with respect to dependence, that is, $i \prec^{+} j \Rightarrow i<j$. Here, $\prec^{+}$denotes the transitive closure of the dependence relation $\prec$. The vertices in $X(Z ; Y)$ are referred to as minimal or independent (intermediate; maximal or dependent) vertices.

The objective is to transform the program that implements $F$ into one that computes the Jacobian matrix (or Jacobian)

$$
F^{\prime}=F^{\prime}\left(\mathbf{x}_{0}\right)=\left(\frac{\partial y_{i}}{\partial x_{j}}\left(\mathbf{x}_{0}\right)\right)_{i=1, \ldots, m, j=1, \ldots, n}
$$

of $F$ with respect to the $n$ inputs for a given argument $\mathbf{x}_{0}$ such that a minimal number of scalar fused multiply-add floatingpoint operations (fmas) are performed. Once numerical values have been computed for all local partial derivatives, scalar floating-point multiplications and additions are the only arithmetic operations required to accumulate $F^{\prime}$. The accumulation of $F^{\prime}$ can be regarded as an elimination procedure in the c-graph $G$ of $F$, as introduced in [1]. The original c-graph is transformed into a subgraph of the directed complete bipartite graph $K_{n, m}$ such that the labels on the remaining edges are exactly the nonzero elements of the Jacobian. The result of this transformation applied to the c-graph from Figure 1 is displayed in Figure 2.

In this paper we develop heuristics for eliminating vertices and edges such that the overall cost of computing $F^{\prime}$ is mini-

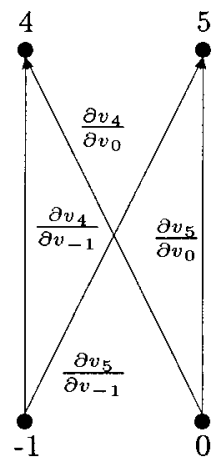

Fig. 2. $F^{\prime}$ mized. A detailed discussion of the corresponding theory can be found in [2]. Here, we introduce only a minimal subset of the framework, in order to focus on new heuristics for vertex and edge elimination in c-graphs. The structure of the paper is as follows. In Section 2 we introduce vertex and edge elimination in c-graphs. Various well known and new Markowitz-type heuristics for both vertex and edge elimination are presented in Section 3. Their properties and performance are discussed in Section 4, and numerical results are presented. Conclusions are drawn in Section 5.

\section{Jacobians by Vertex and Edge Elimination}

The origins of both vertex and edge elimination in c-graphs are in automatic differentiation (AD) [3-6]. This technique modifies the semantics of numerical programs such that derivatives of the underlying vector function can be computed efficiently with machine accuracy. In contrast to divided difference 


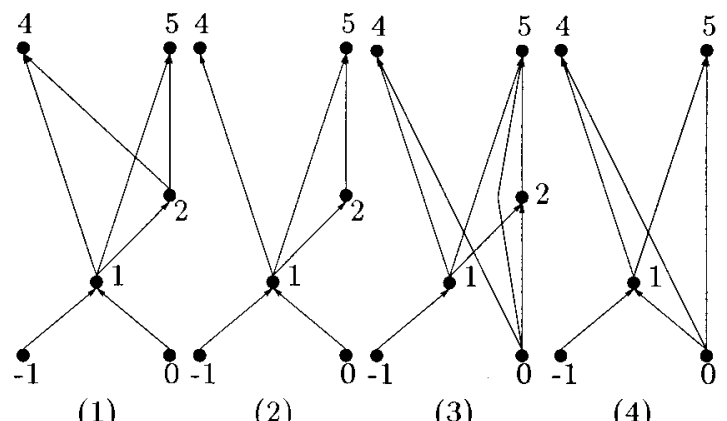

(1)

(2)

(3)

(4)

Fig. 3. Vertex and edge elimination

approximations, AD exploits the chain rule to compute Jacobian times vector products $\dot{\mathbf{y}}=F^{\prime} \dot{\mathbf{x}}$ in forward mode and transposed Jacobian times vector products $\overline{\mathbf{x}}=\left(F^{\prime}\right)^{T} \overline{\mathbf{y}}$ in reverse mode (see [6, Chapter 3] for details). In particular, the Jacobian itself can be obtained at a cost of $n|E|$ in forward mode and $m|E|$ in reverse mode by letting the vectors $\dot{\mathbf{x}}$ and $\overline{\mathbf{y}}$ range over the Cartesian basis vectors in $\mathbb{R}^{n}$ and $\mathbb{R}^{m}$, respectively. The number of edges in the c-graph of $G=(V, E)$ is denoted by $|E|$.

Alternatively, $F^{\prime}$ can be computed by eliminating all intermediate vertices or edges in $G$ as follows. When eliminating an intermediate vertex $j$, new edges are introduced connecting the predecessors of $j$ with its successors. A new edge $(i, k)$ is labeled with the product of the labels of $(j, k)$ and $(i, j)$. Parallel edges are merged, and the corresponding edge labels are added. Finally, $j$ is removed together with its incident edges. The elimination of vertex 3 from the c-graph shown in Figure 1 leads to graph (1) in Figure 3. For example, the new label of $(1,4) \in E$ is equal to $c_{4,1}=c_{4,3} c_{3,1}$. The elimination of vertex 2 in graph (3) leads to graph (4) and, for example, $c_{5,0}=c_{5,0}+c_{5,2} c_{2,0}$. The correctness of the vertex elimination rule is shown in [1]. The number of fmas involved in the elimination of $j$ is referred to as the Markowitz degree of $j$, and it is equal to

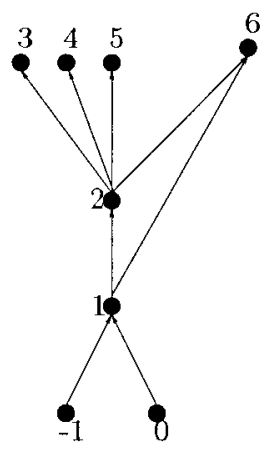

Fig. 4. Lion $\mu_{j}=|\{i: i \prec j\}||\{k: j \prec k\}|$.

The elimination of a vertex $j$ is equivalent to the simultaneous front elimination of all edges leading into it. Similarly, the elimination of $j$ is equivalent to the simultaneous back elimination of all edges emanating from it. An edge $(i, j)$ is front eliminated by connecting $i$ with all successors of $j$. For all successors $k$ of $j$, the new edges $(i, k)$ are labeled with $c_{k, i}=c_{k, j} c_{j, i}$. If $(i, k)$ existed before, then $c_{k, i}=c_{k, i}+c_{k, j} c_{j, i}$. The edge $(i, j)$ is removed after this. The number of fmas required to front eliminate $(i, j)$ is equal to $\{\{k: j \prec k\} \mid$. The front elimination of $(0,1)$ transforms graph $(2)$ into graph $(3)$ in Figure 3 . Analogously, an edge $(j, k)$ 
is back eliminated by connecting all predecessors of $j$ with $k$. For all predecessors $i$ of $j$ the new edge $(i, k)$ is labeled with $c_{k, i}=c_{k, j} c_{j, i}$. Again, the label becomes $c_{k, i}=c_{k, i}+c_{k, j} c_{j, i}$ if $(i, k)$ existed before. Finally, $(j, k)$ is removed. The number of $\mathbf{f m a s}$ required to back eliminate $(j, k)$ is equal to $|\{i: i \prec j\}|$. In Figure 3, graph (2) can be obtained from graph (1) by back elimination of $(2,4)$. Newly generated edges are referred to as fill-in. Absorption takes place whenever two parallel edges are merged.

The set of all valid vertex eliminations is contained within the set of all valid edge eliminations. Hence, the optimal vertex elimination sequence is contained within the set of all edge elimination sequences, and thus the optimal edge elimination sequence performs at most the same number of arithmetic operations as the optimal vertex elimination sequence. The lion graph [7] displayed in Figure 4 represents one example where the optimal edge elimination sequence involves fewer operations than does the optimal vertex elimination sequence. Both elimination sequences require $4+8=12$ fmas. The back elimination of $(2,6)$ followed by the elimination of 1 and 2 reduces this number by one. Refer to [2] for a more detailed investigation of this vertex-edge discrepancy. It is the motivation for introducing edge elimination in addition to the conceptually much easier vertex elimination method.

We assume the chain rule to be associative over the floating point numbers. In other words, the numerical values of the entries in $F^{\prime}$ do not depend on the order in which the intermediate vertices or edges are eliminated. However, the computational cost varies. In Figure 1, for example, the vertex elimination sequence $[1,2,3]$ performs $4+2+4=10$ fmas. The reader may wish to verify that $[2,1,3]$ takes only $1+2+4=7$ fmas. The cost of computing $F^{\prime}$ by using either the forward or the reverse mode of AD is $2 \cdot 7=14$. Even on this small example, the operations count can be reduced by a factor of two. This is the primary motivation for investigating heuristics for vertex and edge elimination in Section 3.

\section{Heuristics}

Both the vertex and edge elimination problems in c-graphs are conjectured to be NP-complete $[8,1,7]$. No polynomial algorithm is known for solving them exactly. The problem of minimizing the fill-in under vertex elimination was shown to be NP-complete by Herley [9] in an unpublished adaption of a note by Gilbert [10] on a result by Rose and Tarjan [11] about vertex elimination techniques for solving sparse linear systems. So far, it remains unclear whether the same is true for edge and face [2] elimination.

In the following we write $H(G)=i$ whenever the application of a heuristic $H$ to a c-graph $G$ gives the vertex $i$ as a result. An analogous notation is used for edge elimination heuristics. All heuristics $H$ are defined such that $|H(G)|=1$, that is, the result of applying $H$ to $G$ should contain a single vertex or edge. 


\subsection{Forward Vertex and Edge Elimination}

The forward vertex elimination mode $\mathrm{FM}_{v}$ eliminates the intermediate vertices in ascending order with respect to their indices, that is, $\mathbf{F M}_{v}(G)=j \Leftrightarrow \forall i \in$ $V: j \leq i$. The same idea can be applied to edge elimination. For reasons of consistency, we require the forward edge elimination mode $\mathrm{FM}_{e}$ to have the same computational cost as $\mathbf{F M}_{v}$. This can be achieved by pure back edge elimination sequences in lexicographical order or by pure front edge elimination sequences in switched lexicographical order. For example, the latter can be written as $\mathrm{FM}_{e}(G)=(i, j)_{f}$ if

$$
(i, j) \in E \cap(V \times Z) \wedge \forall(k, l) \in E \cap(V \times Z): j \leq l \vee j=l \wedge i \leq k .
$$

The fact that an edge $(i, j)$ is front (back) eliminated is denoted by $(i, j)_{f}\left((i, j)_{b}\right)$. Note that the set of edges that can be front eliminated is restricted to those having an intermediate vertex as target.

\subsection{Reverse Vertex and Edge Elimination}

In reverse vertex elimination mode the intermediate vertices are eliminated in descending order starting with $p$, that is, $\operatorname{RM}_{v}(G)=j \Leftrightarrow \forall i \in V: j \geq i$. The extension to edge elimination sequences is similar to $\mathbf{F M}$, for example, $\operatorname{RM}_{e}(G)=$ $(i, j)_{f}$ if

$$
(i, j) \in E \cap(Z \times V) \wedge \forall(k, l) \in E \cap(Z \times V): j \geq l \vee j=l \wedge i \geq k .
$$

To ensure uniqueness of the result, one must combine all heuristics with a tiebreaking criterion or even a hierarchy of tiebreakers. We use either FM or RM (for vertices or edges, depending on the context) as the "bottom line," since we always have $|\operatorname{FM}(G)|=1$ as well as $|\operatorname{RM}(G)|=1$.

\subsection{Lowest Markowitz}

The lowest Markowitz (LM) degree-first heuristic was introduced in [1]. It was motivated by a similar idea from the theory of direct methods for solving sparse linear systems.

Heuristic $1 L M_{v}(G)=j$ if

$$
\forall i \in V: \mu_{j} \leq \mu_{i} \wedge \forall i \in V^{\prime}, V^{\prime}=\left\{k \in V: \mu_{j}=\mu_{k}\right\}: j=R M_{v}\left(G^{\prime}\right),
$$

where $G^{\prime}$ is the subgraph of $G$ that is induced by $V^{\prime}$.

$G^{\prime}=\left(V^{\prime}, E^{\prime}\right)$ is said to be induced by a vertex set $V^{\prime} \subseteq V$ if $E^{\prime}=\{(i, j): i \in$ $\left.V^{\prime} \wedge j \in V^{\prime}\right\}$. In general, we always have the choice to use either $\mathbf{F M}_{v}$ or $\mathbf{R M}_{v}$ as the ultimate tiebreaker to make the result of a heuristic unique. This choice, however, may affect the performance of the heuristic significantly. 
As for $\mathrm{FM}_{v}$ and $\mathrm{RM}_{v}$, the computational effort of $\mathrm{LM}_{v}$ is constant per vertex and per iteration. The number of iterations is equal to the number of intermediate vertices $p$, and the vertices taken into account are the intermediate vertices that have not been eliminated yet. Hence, the maximal overall effort is $p(p+1) / 2 \in$ $\mathcal{O}\left(|V|^{2}\right)$.

The Markowitz degree of $(i, j) \in E$ is defined as $\min (|\{k: j \prec k\}|, \mid\{k: k \prec$ $i\} \mid)$. The edge $(i, j)$ is front eliminated if $|\{k: j \prec k\}| \leq|\{k: k \prec i\}|$. Otherwise, it is back eliminated. The lowest-Markowitz heuristic for edge elimination $\mathbf{L M}_{\boldsymbol{e}}$ is defined analogous to Heuristic 1. Its complexity, however, also depends on the fill-in that is generated. Although, the termination of edge elimination was shown in [7], it remains unclear whether the cost of edge elimination sequences is still polynomial in the worst case. This question is the subject of ongoing research.

\subsection{Lowest Relative Markowitz}

The lowest relative Markowitz (LRM) degree-first heuristic is an extension of LM. It was introduced in [12]. Let $\iota_{j}=\left|\left\{k: k \in X \wedge k \prec^{+} j\right\}\right|, \delta_{j}=\mid\{k: k \in$ $\left.Y \wedge j \prec^{+} k\right\} \mid$, and $\hat{\mu}_{j}=\mu_{j}-\iota_{j} \cdot \delta_{j}$. The relative Markowitz degree is defined as the difference between the Markowitz degree and the dependence degree $\iota_{j} \cdot \delta_{j}$ of the vertex $j$. The idea is to maximize the dependence degree while, at the same time, minimizing $\mu_{j}$. This heuristic is usually combined with $\mathrm{LM}_{v}$ as tiebreaker.

Heuristic $2 \operatorname{LRM}_{v}(G)=j$ if

$$
\forall i \in V: \hat{\mu}_{j} \leq \hat{\mu}_{i} \wedge \forall i \in V^{\prime}, V^{\prime}=\left\{k \in V: \hat{\mu}_{j}=\hat{\mu}_{k}\right\}: j=L M_{v}\left(G^{\prime}\right)
$$

where $G^{\prime}$ is the subgraph of $G$ that is induced by $V^{\prime}$.

See [12] for further details.

To formulate LRM for edge elimination, we set

$$
\hat{\mu}((i, j))=\min \left(|\{k: j \prec k\}|-\delta_{j},|\{k: k \prec i\}|-\iota_{i}\right) .
$$

As for $\mathrm{LM}_{e}$, an edge $(i, j)$ is front eliminated if $|\{k: j \prec k\}|-\delta_{j} \leq \mid\{k: k \prec$ $i\} \mid-\iota_{i}$ and else back eliminated. Thus, $\mathbf{L R M}_{e}$ can be derived immediately from Heuristic 2.

\subsection{Maximal Overall Markowitz Degree Reduction}

The maximal overall markowitz degree reduction (MOMR) heuristic represents the global pendant to LM. It considers the effect of the elimination of a vertex on the Markowitz degrees of its neighbors instead of its own Markowitz degree. Therefore we define the overall Markowitz degree of a c-graph $G=(V, E)$ as $M=M(G)=\sum_{i \in V} \mu_{i}$. The overall Markowitz degree reduction of a vertex $i \in V$ is defined as $\mu_{j}^{-}=M(G)-M(G-j)$, where $G-j$ is the c-graph after the elimination of $j$. 
Heuristic $3 \operatorname{MOMR}_{v}(G)=j$ if

$$
\forall i \in V: \mu_{i}^{-} \leq \mu_{j}^{-} \wedge \forall i \in V^{\prime}, V^{\prime}=\left\{k \in V: \mu_{j}^{-}=\mu_{k}^{-}\right\}: j=L M_{v}\left(G^{\prime}\right) \text {, }
$$

where $G^{\prime}$ is the subgraph of $G$ that is induced by $V^{\prime}$.

Initially, $\mathbf{M O M R}_{v}$ attempts to reduce the Markowitz degrees of its neighbors as much as possible, thus making use of absorption while trying to avoid excessive fill-in. Only as a second step does it take the vertex's own Markowitz degree into account. MOMR $_{v}$ reduces the cost of eliminating the remaining vertices as much as possible.

To calculate the Markowitz degree reduction of $k$ caused by the elimination of some $j \prec k$, one must compute the difference of their respective predecessor sets. Similarly, the successor sets must be considered if $k \prec j$. Hence, MOMR $_{v}$ involves at most $\mathcal{O}\left(a^{2}|V|^{2}\right)$ operations, where $a$ is the average number of predecessors (or successors) per vertex over all elimination steps.

The formulation of MOMR for edge elimination is straightforward by comparing the overall Markowitz degree of $G$ before and after the front and back elimination of an edge.

\subsection{Lowest Markowitz Minimal Damage}

This lowest Markowitz minimal damage (LMMD) heuristic combines LM and MOMR. The effect of MOMR is twofold. On one hand, it enforces the elimination of vertices that reduce the Markowitz degrees of their neighbors maximally (or at least do not increase them too much). On the other hand, it implicitly prefers vertices with high Markowitz degrees, and hence incurs a high elimination cost.

The idea behind LMMD is to look for vertices with low Markowitz degrees that cause a minimal increase of the Markowitz degree on other vertices (minimal damage). If the overall Markowitz degree without considering $j \in V$ of a c-graph $G=(V, E)$ is defined as $\hat{M}_{j}(G)=\sum_{i \neq j} \mu_{i}$, then the damage caused by the elimination of $j$ is set to $d_{j}=M(G-j)-\hat{M}_{j}(G)$. In order to increase the flexibility of LMMD, the damage is scaled with a weight $w$.

Heuristic $4 L M M D_{v}(w, G)=j$ if $\forall i \in V \mu_{j}+w d_{j} \leq \mu_{i}+w d_{i}$ and $\forall i \in$ $V^{\prime}, V^{\prime}=\left\{k \in V: \mu_{j}+w d_{j}=\mu_{k}+w d_{k}\right\}: j=R M_{v}\left(V^{\prime}\right)$.

W.l.o.g., we choose $\mathrm{RM}_{v}$ as a tiebreaker. Other criteria can be used as well; however, especially $\mathbf{L M}_{v}$ as tiebreaker would be somewhat redundant because of the already present implicit orientation toward the lowest Markowitz degree. Choosing a small factor $w$ focuses on the Markowitz degree of the vertex and reduces the value of the damage to a tiebreaker. Large values, on the other hand, emphasize the Markowitz degree reduction of the neighboring vertices and degrade the current degree to a secondary criterion. The computational effort is identical to that of MOMR .

Again, the formulation of $\mathrm{LMMD}_{e}$ is analogous to Heuristic 4, when considering the damage caused by front and back edge elimination, respectively. 
Table 1. Elimination Costs for Graphs from Structured Random C-Graph Generator

\begin{tabular}{|rrr|rr|rr|rr|rr|r|}
\hline$n$ & $p$ & $m$ & \multicolumn{2}{|r|}{ FM RM } & LM1 LM2 & LRM1 & LRM2 & MOMR1 & MOMR2 & LMMD \\
\hline 3 & 64 & 2 & 108 & 87 & 89 & 77 & 93 & 86 & 83 & 81 & 77 \\
& & 108 & 87 & 104 & 87 & 106 & 86 & 83 & 81 & 78 \\
\hline 3 & 206 & 3 & 638 & 41. & 374 & 316 & 392 & 389 & 337 & 342 & 312 \\
& & 638 & 418 & 553 & 351 & 448 & 417 & 333 & 336 & 334 \\
\hline \multirow{2}{10}{} & 236 & 10 & 1216 & 609 & 480 & 425 & 520 & 499 & 434 & 441 & 417 \\
& & 1216 & 609 & 666 & 462 & 507 & 467 & 448 & 454 & 447 \\
\hline 10 & 243 & 3 & 1567 & 435 & 468 & 369 & 448 & 430 & 411 & 406 & 367 \\
& & 1567 & 435 & 837 & 392 & 813 & 418 & 428 & 398 & 380 \\
\hline 10 & 245 & 1 & 1605 & 349 & 454 & 326 & 461 & 430 & 356 & 357 & 325 \\
& & 1605 & 349 & 801 & 349 & 614 & 352 & 339 & 339 & 332 \\
\hline 3 & 236 & 10 & 718 & 649 & 463 & 420 & 526 & 519 & 435 & 462 & 415 \\
& & 718 & 649 & 646 & 460 & 669 & 488 & 440 & 459 & 453 \\
\hline 1 & 236 & 10 & 337 & 651 & 423 & 373 & 444 & 432 & 342 & 394 & 356 \\
& & 337 & 651 & 337 & 404 & 742 & 426 & 334 & 368 & 352 \\
\hline
\end{tabular}

\section{Numerical Results}

The generation of c-graphs from real-world application programs requires a fully functional compiler front-end to transform the program into an abstract syntax tree. We are using the tool EliAD [13] to perform this task. The heuristics were applied to the accumulation of the Jacobian of Roe's numerical flux [14], coating thickness standardization, combustion of propane, full formulation, human heart dipole, and flow in a channel. All but the Roe flux are part of the MINPACK2 test problem collection [15]. The computational graphs were generated by unrolling all loops for a given input.

Unfortunately, the structure of the resulting graphs is not very complicated, and it shows a strong regularity. The LM heuristic works well in all these cases. Although, this feature is likely to be exhibited by many real-world simulation codes, the corresponding graphs are not very useful for studying the effect of heuristics on the number of fmas that are required to accumulate the corresponding Jacobian. Therefore, we have developed a random c-graph generator [16]. It allows us to check the behavior of various heuristics on a large number of c-graphs of varying sizes.

In Table 1 we have listed the results obtained by applying the heuristics discussed in this paper to a number of randomly generated c-graphs. For a given graph, the first row shows the results of the heuristics for vertex elimination, and the second row shows the values obtained by the corresponding heuristic for edge elimination. We observe that MOMR and LMMD show the most consistent behavior, while LMMD delivers the best results in most cases. RM outperforms FM if there are fewer outputs than inputs. FM delivers better results if the number of outputs exceeds the number of inputs. If there are as many outputs as there are inputs, then RM seems to perform better. A likely reason is that for the 
intermediate vertices the number of successors is arbitrary, whereas the number of predecessors is limited by two. LM appears to be a reasonable compromise for a large number of c-graphs. Typically, it performs almost as well as the best heuristic.

An important goal of the research that led to this paper was the investigation of Markowitz degree-based heuristics for edge elimination. We expected our results to be improved by simply reformulating the ideas behind the vertex elimination heuristics in the context of edge elimination. The opposite appears to be the case. Apart from the last example, none of the edge elimination heuristics delivers better results than the best vertex elimination heuristic. Apparently, the heuristics are influenced negatively by the considerably widened search space. We conclude that the implicit locality of eliminating all edges incident to a vertex is usually more beneficial than the higher degree of freedom when considering the heuristics that are discussed here.

\section{Conclusion}

Both MOMR and LMMD represent good choices for Markowitz-based vertex elimination heuristics. On a representative test set (a subset of which was presented here) they exhibit a consistent behavior in terms of the quality of the elimination sequences that were generated. In particular, they exhibit an increased robustness with respect to the choice of different tiebreaking criteria. Similar ideas were applied to edge elimination with no noticeable improvement in the cost of the elimination sequences generated. We conclude that different criteria must be developed in order to exploit the power of edge elimination for accumulating Jacobians. How edge elimination sequences can reduce the cost of vertex elimination sequences is the subject of ongoing research.

Similar to the approach taken in [17], we are implementing logarithmic simulated annealing algorithms [18] for edge elimination. We hope to observe discrepancies between the optimal vertex and edge elimination sequences for real-world applications. From the structure of such problems we expect to learn more about suitable criteria for edge elimination heuristics. Furthermore, a detailed investigation of the energy landscape of the various combinatorial optimization problems arising in Jacobian computation will allow us to gain insight into potential improvements. Finally, we conclude that, in view of our promising results, robust heuristics for accumulating Jacobians efficiently should become a key feature of software tools for automatic differentiation.

\section{Acknowledgments}

This research is supported by the UK's Engineering and Physical Sciences Research Council under grant GR/R/38101/01.

Naumann is supported by the Mathematical, Information, and Computational Sciences Division subprogram of the Office of Advanced Scientific Computing Research, U.S. Department of Energy, under Contract W-31-109-ENG-38. 


\section{References}

1. Griewank, A., Reese, S.: On the calculation of Jacobian matrices by the Markovitz rule. In: [5]. (1991) 126-135

2. Naumann, U.: Optimal accumulation of Jacobians by elimination methods on the dual computational graph. Mathematical Programming (2002) to appear.

3. Berz, M., Bischof, C., Corliss, G., Griewank, A., eds.: Computational Differentiation: Techniques, Applications, and Tools, Philadelphia, SIAM (1996)

4. Corliss, G., Faure, C., Griewank, A., Hascoet, L., Naumann, U., eds.: Automatic Differentiation of Algorithms - from Simulation to Optimization, New York, Springer (2002)

5. Corliss, G., Griewank, A., eds.: Automatic Differentiation: Theory, Implementation, and Application, Philadelphia, SIAM (1991)

6. Griewank, A.: Evaluating Derivatives. Principles and Techniques of Algorithmic Differentiation. Number 19 in Frontiers in Applied Mathematics. SIAM, Philadelphia (2000)

7. Naumann, U.: Efficient Calculation of Jacobian Matrices by Optimized Application of the Chain Rule to Computational Graphs. PhD thesis, Technical University Dresden (1999)

8. Bischof, C., Haghighat, M.: Hierarchical approaches to automatic differentiation. In: [3]. (1996) 82-94

9. Herley, K.: A note on the NP-completeness of optimum Jacobian accumulation by vertex elimination. Presentation at: Theory Institute on Combinatorial Challenges in Computational Differentiation (1993)

10. Gilbert, J.: A note on the NP-completeness of vertex elimination on directed graphs. J. Alg. Disc. Meth. 1 (1980) 292-294

11. Rose, D., Tarjan, R.: Algorithmic aspects of vertex elimination on directed graphs. J. Appl. Math. 34 (1978) 176-197

12. Naumann, U.: An enhanced Markowitz rule for accumulating Jacobians efficiently. In Mikula, K., ed.: ALGORITHMY'2000 Conference on Scientific Computing, Slovak University of Technology, Bratislava, Slovakia (2000) 320-329

13. Tadjouddine, M., Forth, S., Pryce, J., Reid, J.: Performance issues for vertex elimination methods in computing Jacobians using Automatic Differentiation. In: Proceedings of the ICCS 2000 Conference. Volume 2330 of Springer LNCS. (2002) 1077-1086

14. Roe, P.: Approximating Riemann solvers, parameter vectors, and difference schemes. J. Comp. Physics (1981) 357-372

15. Averik, B., Carter, R., Moré, J.: The MINPACK-2 test problem collection (preliminary version). Technical Report 150, Mathematical and Computer Science Division, Argonne National Laboratory (1991)

16. Albrecht, A., Gottschling, P., Naumann, U.: Logarithmic simulated annealing for optimal derivative code. Technical Report 372, University of Hertfordshire (2002)

17. Naumann, U., Gottschling, P.: Prospects for simulated annealing in automatic differentiation. In Steinhöfel, K., ed.: SAGA 2002 - Stochastic Algorithms, Foundations and Applications. Volume 2264 of LNCS., Springer, Berlin (2001) 131-144

18. Albrecht, A., Wong, C.: On logarithmic simulated annealing. In van Leeuwen, J., Watanabe, O., Hagiya, M., Mosses, P., eds.: Proc. IFIP International Conference on Theoretical Computer Science. LNCS, Springer (2000) 